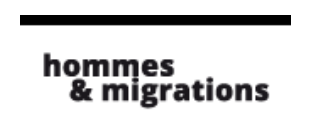

Hommes \& migrations

Revue française de référence sur les dynamiques

migratoires

$1293 \mid 2011$

L'immigration dans les musées

\title{
La place de l'art contemporain à la Cité
}

Entretien avec Jacques Toubon, président du conseil d'orientation de la Cité nationale de l'histoire de l'immigration

\section{Isabelle Renard et Marie Poinsot}

\section{(2) OpenEdition}

\section{Journals}

Édition électronique

URL : http://journals.openedition.org/hommesmigrations/497

DOI : 10.4000/hommesmigrations.497

ISSN : 2262-3353

Éditeur

Musée national de l'histoire de l'immigration

Édition imprimée

Date de publication : 1 septembre 2011

Pagination : 22-26

ISSN : 1142-852X

Référence électronique

Isabelle Renard et Marie Poinsot, «La place de l'art contemporain à la Cité », Hommes \& migrations [En ligne], 1293 | 2011, mis en ligne le 31 décembre 2013, consulté le 19 avril 2019. URL : http:// journals.openedition.org/hommesmigrations/497 ; DOI : 10.4000/hommesmigrations.497 


\section{La place de l’art contemporain à la Cité Entretien avec Jacques Toubon, président du conseil d'orientation de la Cité nationale de l'histoire de l'immigration}

Par Isabelle Renard, chargée de mission pour la collection d'art contemporain du Musée de l'histoire et des cultures de l'immigration, $\mathrm{CNHI}$ et Marie Poinsot

\section{H\&M : Dans quel contexte avez-vous mis en place les collections du mu- sée d’histoire et des cultures de l'immigration?}

Jacques Toubon : En 2007, avec l'ouverture de son exposition permanente "Repères", la Cité nationale de l'histoire de l'immigration a tenté de proposer une forme muséale originale, à la croisée du musée d'histoire, du musée de société et du musée d'art. Dès le départ, les collections ont abordé ensemble le passé, le présent et le futur de l'immigration. La Cité se situe à l'intersection du temps historique et de l'espace de la société contemporaine. Faire connaître et reconnaître l'histoire de l'immigration pour changer le regard sur l'immigration nécessite de conjuguer trois approches. L'approche historique, avec des sources contextualisées par les historiens les plus compétents ; l'approche anthropologique, constituée par des témoignages individuels ou familiaux, des dons d'objets ou de documents, et l'approche artistique, avec des ceuvres majeures des dernières décennies qui composent une collection d'art contemporain singulière. C'est une partie de cette collection d'art contemporain que l'exposition J'ai deux amours présente de novembre 2011 à juin 2012.

Aujourd'hui, l'art contemporain est présent dans toutes les institutions culturelles, dont l'une des missions est de soutenir les artistes et la création. La Cité a considéré dès sa constitution que la collection permanente devait rassembler des sources multiples, différentes et toutes aussi importantes, qu'il s'agisse de documents historiques, d'objets personnels ou d'ceuvres d'art. 
H\&M : Quelle définition pourriez-vous donner de "contemporain" ? Pour le philosophe italien Giorgio Agamben, le contemporain est "celui qui reçoit en plein visage le faisceau des ténèbres de son temps ${ }^{(1) \text { g'. }}$.

J. T. : L'art contemporain se définit d'abord en termes de temporalité. Il permet d'être en présence à la fois de l'ceuvre et de l'artiste. Depuis une trentaine d'années, le "contemporain" désigne aussi ce qui est "étendu au monde tel qu'il est". Il permet de saisir le monde dans toute son extension, tel qu'il est rendu à travers l'expression artistique. Cela représente pour le public la possibilité de l'échange, du voyage. Pour nous qui nous intéressons à l'immigration, l'art contemporain renvoie à tous les enjeux liés à cette question. Pendant longtemps, l'art contemporain signifiait "l'art des gens du quartier d'à côté". Par ailleurs, même si toute époque a eu son art contemporain, aujourd'hui plus que jamais, du fait de la mobilité et de l'ouverture de la société, de la médiatisation, l'art contemporain est le fait d'artistes en mesure de prendre position, de bousculer et bouleverser la société dans laquelle ils vivent. On pourrait dire la même chose de Delacroix et de Rembrandt à leur époque, mais à un moindre degré. Ainsi, il est clair que le contemporain aujourd'hui est vivant, mondial et engagé, ou du moins concerné par la réalité. Cela va plus loin que le mouvement qu'on a appelé des peintres témoins de leur temps, qui, à l'époque, étaient mobilisés du côté du Parti communiste.

L'art contemporain est évidemment en relation avec notre travail et notre domaine d'action. Certes, des artistes décédés méritent néanmoins de figurer dans les expositions d'art contemporain, car le contemporain absolu consisterait à ne montrer dans les expositions que des ceuvres réalisées en 2011, ce qui serait bien entendu absurde. Il y a une période contemporaine couverte par des artistes vivants ou décédés, dont les ceuvres peuvent être définies comme contemporaines, et qui se distinguent de celles d'artistes que l'on peut définir comme modernes. C'est une distinction nette.

\section{H\&M : Quelle est la relation entre histoire et art contemporain, dans le contexte de la Cité ?}

J. T. : L'un des apports de l'art est le récit, transmis de manière explicite ou non à travers des ceuvres mobilisant la photo, la peinture et la vidéo. Par définition, le récit est aussi de l'histoire. Sur notre sujet en particulier, les ceuvres contemporaines racontent des histoires, singulières ou collectives. Elles donnent un point de vue et un récit subjectifs qui peuvent être en accord ou en contradiction avec le récit scientifique. Elles peuvent soit corroborer, renforcer, voire démontrer le récit des historiens, soit le relativiser ou même le démonter. Elles facilitent le passage de l'histoire à l'Histoire. 
Le deuxième apport de l'art contemporain est de susciter l'émotion. Les ceuvres provoquent un choc émotionnel, des réminiscences, ou au contraire la contestation. Elles complètent la collection anthropologique du musée composée de témoignages et de dons personnels qui confèrent déjà un visage humain à l'histoire de l'immigration.

La fonction de récit, qui renforce l'aspect scientifique ou le met en doute, le relativise, me paraît tout à fait fondamentale. Par exemple, on essaye de travailler sur la question des religions.

L'ceuvre de Kader Attia, présentée dans l'exposition, crée, à ce propos, une grande ambiguïté autour de l'objet des règles religieuses : Que signifie le terme "hallal" ? Pour l'artiste, son ceuvre est une façon de détourner l'un des préceptes de la religion musulmane, montrant qu'en désignant une forme de la société de consommation parmi d'autres, le terme s'est finalement progressivement vidé de son sens. De ce point de vue, il peut corroborer un discours sur la présumée modernisation ou laïcisation de l'islam. Cela peut également être une illustration des conflits qui existent au sein de l'islam, ou encore interpeller un public non musulman qui interpréterait cela comme le signe de la création d'une société hallal, ces produits étant de plus en plus nombreux. À travers les questions qu'elle suscite, cette ceuvre constitue un apport indiscutable au dispositif muséal de la Cité, et notamment à la partie consacrée à la religion : est-ce le signe d'une forme d'évolution vers la neutralité et la laïcité ?

\section{H\&M : Pour l’artiste, l'œuvre souligne la difficile équation entre le désir d'appartenance à une société moderne occidentale et la préservation de valeurs traditionnelles. Mais elle pose aussi la question de la société de consommation, de la norme de vie érigée par le capitalisme : "Je consomme donc je suis."}

J. T. : On revient ici au propos initial : l'ceuvre contemporaine peut dévoiler son mystère plus facilement que les autres, grâce à un dialogue possible avec l'artiste. Très peu d'artistes ont décrit leurs ceuvres et les historiens de l'art nous expliquent le parcours des ceuvres plus souvent que celui des artistes. Avec l'artiste vivant, la relation entre l'ceuvre et le public est différente : le regard sur la portée sociale et politique est plus ouvert. Je crois que dans le cadre d'une institution comme la nôtre que doit se faire un travail de médiation et d'explication, non pas parce que cet art serait plus compliqué qu'un autre, mais parce que nous pouvons faire un travail de ce type, étant en contact avec les artistes. Par exemple, dans l'exposition J'ai deux amours, les cartels explicatifs donnent la parole aux artistes et complètent la présentation des ceuvres. 


\section{H\&M : On peut se demander si l'œuvre d'art est illustration d'un propos ou lieu d'interrogation.}

J. T. : Les ceuvres contemporaines interrogent la société. Ainsi, le visiteur n'est pas seulement un spectateur, mais aussi un acteur. À travers une ceuvre qui le touche, le fait réagir, le provoque, se crée avec le visiteur un lien particulier. Il me paraît important de souligner cette multiplicité de sens propre à l'art contemporain, qui met également en valeur la question de la relation entre le récit et l'émotion. C'est de cette façon que l'œuvre vivante acquiert sa complexité.

Il y a deux manières de voir les ceuvres contemporaines : l'une est purement esthétique, il s'agit de comprendre l'effet sur le spectateur. L'autre concerne le sens, le récit de l'ceuvre elle-même, qui peut être multiple et polysémique. Il faut à la fois respecter la première réaction du public, son l'émotion ("Est-ce que cela me plaît ? Est-ce que cela est beau ou laid pour moi ?") et le deuxième temps, celui de l'explication. À ce stade, 
se pose la question de savoir s'il faut proposer au visiteur une quelconque forme d'explication : comment trouver le moyen de répondre à la quête de sens du visiteur, à sa réaction, interrogation ou déception, sans lui imposer une lecture déterminée ? Personnellement, lorsque je suis confronté à une ceuvre d'art, surtout contemporaine, je n'aime pas que l'on m'impose trop de littérature. Si je souhaite trouver du sens audelà de mon émotion esthétique première, j'apprécie que l'on me fournisse des éléments de compréhension, mais je ne veux pas que l'on m'impose de comprendre ce que l'ceuvre dit, si elle me satisfait déjà d'un point de vue purement esthétique.

L'écho de l'ceuvre peut être infini et implique de la part de la Cité un travail de médiation complexe entre les ceuvres qui interpellent et parfois bousculent le public. Il y a là un équilibre très subtil à trouver entre une demande qu'il faut comprendre, et à laquelle il faut répondre, et une absence de demande qu'il faut respecter.

\section{H\&M : La CNHI a choisi, dans un premier temps, pour son exposition permanente des œuvres dont le but était d'éclairer l'histoire de l'immi- gration en France sous ses différents aspects. Depuis quelque temps, elle s'autorise à choisir des œuvres plus métaphoriques, qui dépassent l'objet de la Cité et le cadre français, et s'adressent à l'universel. Pensez- vous que cette orientation soit juste ?}

J. T. : Grâce à une politique d'acquisition ambitieuse dès la création du musée, la Cité a constitué une collection d'œeuvres contemporaines remarquable, en cohérence avec son objet et sa mission, et doit poursuivre sur cette voie. Car nombre d'ceuvres contemporaines jettent un regard universel sur les questions liées aux migrations et viennent éclairer d'un jour nouveau le propos de la Cité.

Le fait que la Cité acquière des ceuvres décalées par rapport à l'histoire de l'immigration en France me parait souhaitable, à condition que l'on puisse faire l'aller-retour entre les enjeux universels qu'elles peuvent représenter et les problématiques propres à la mission de la Cité, c'est-à-dire l'histoire passée et le présent de l'immigration en France. En revanche, des cuvres qui seraient liées à un contexte géographique précis me paraîtraient relever d'autres institutions que la nôtre. Le Musée doit poursuivre cette politique qui lui a permis en moins de cinq années de constituer une collection excellente du point de vue de l'art et cohérente avec son objet et sa mission.

\section{Notes}

1. Giorgio Agamben, Qu'est-ce que le contemporain? Paris, Payot \& Rivages, 2008. 